\title{
On the propagation of analyticity of solutions of convolution equations
}

\author{
By \\ Takahiro KawAI
}

(Received June 28, 1972)

The purpose of this note is to prove a theorem on the propagation of the real analyticity of solutions of convolution equations by the simplest way in its nature, i. e., avoiding the explicit use of the $a$ priori estimate for convolution operators. (Cf. Kawai [2] §5, where the analogous problem is treated for linear differential operators (of infinite order) with constant coefficients by combining the theory of Fourier hyperfunctions with inequalities for holomorphic functions. See also Kawai [1] Theorem 3.2.1. There a related problem is treated for convolution operators and the method of its proof can be used for our purpose, though we do not employ it here.) The method of the proof given below is essentially the same as that employed in the previous note (Kawai [3]), that is, our proof essentially relies on the theory of microfunctions, especially on the notion of integration along fiber of microfunctions (Sato [5] §6. See also Sato, Kawai and Kashiware [7] Chapter I). Note that the convolution product $\mu * \nu$ of two hyperfunctions $\mu$ and $\nu$ defined on $\boldsymbol{R}^{n}$, one of which has compact support, is nothing but the following special kind of integral along fiber of a hyperfunction (Sato [5] § 10)

$$
\int \mu(x-y) \nu(y) d y=\int \mu(y) \nu(x-y) d y,
$$

which is consistent with that of a microfunction. (Sato [6] $\S 6$ )

We first recall the following condition $(S)$ on the convolution 
operator $S *$, where $S$ is a hyperfunction with compact support in $\boldsymbol{R}^{n}$.

Definition. (Cf. Kawai [2] Definition 4.4.1.) Let $J(\zeta)$ be the Fourier transform of $S(x)$, i. e., $\langle S(x), \exp (\sqrt{-1}\langle x, \zeta\rangle)\rangle$. Then the convolution operator $S *$ is said to satisfy condition $(S)$ when the following condition holds.

For any positive $\varepsilon$ there exists a constant $N_{\varepsilon}$ which satisfies the following:

For any $\xi \in \boldsymbol{R}^{n}$ with $|\xi|>N_{\varepsilon}$ we can find some $\zeta \in C^{n}$ satisfying

$$
|\xi-\zeta|<\varepsilon|\xi|
$$

and

$$
|J(\zeta)|>\exp (-\varepsilon|\xi|) \text {. }
$$

Remark. This condition on the convolution operator $S *$ is a very generous one. For example, condition $(S)$ is always satisfied if $S$ a distribution with compact support (Kawai [2] p. 499), or more generally, if $S$ is a ultradistribution with compact support in the sense of Roumieu [4]. It is also easily verified that the convolution operator $S *$ satisfies condition $(S)$ if $\operatorname{supp} S=\{0\}$, i. e., if $S *$ reduces to a linear differential operator of infinite order with constant coefficients. (Cf. Kawai [2] Lemma 4.1.5.) In the latter case the theorem which we present here follows from Theorem 5.1.1. in Kawai [2].

Now our result is the following

Theorem. Let $S *$ be a convolution operator satisfying condition $(S)$. Let $u$ be a hyperfunction defined on $\boldsymbol{R}^{n}$ which satisfies

(3) $S * u$ is real analytic on $\boldsymbol{R}^{n}$

and

(4) $u$ is real analytic outside a compact set $K$.

Then $u$ is real analytic on $\boldsymbol{R}^{n}$.

Proof. To begin with, we recall the fact that condition $(S)$ 
assures the solvability of convolution equation on $\boldsymbol{R}^{n}$

$$
S * v=f \text {, }
$$

i. e., for any hyperfunction $f(x)$ defined on $\boldsymbol{R}^{n}$ we can find a hyperfunction $v(x)$ which satisfies (5) under condition $(S)$. (Kawai [2] Theorem 4.2.4.) Therefore we can find a hyperfunction $E(x)$ which satisfies

$$
S * E=\boldsymbol{\delta},
$$

where $\delta$ denotes Dirac's delta function.

Let $u(x)$ be a hyperfunction which satisfies (3) and (4). Denoting supp $S(x)$ by L we investigate S. S. $(S(w-x) E(x-y) u(y))$ and S.S. $(S(w-x) E(y) u(x-y))$. Here for hyperfunction $\mu$ on a real analytic manifold $M$ we denote by S.S. $\mu$ the support of $\operatorname{sp}(\mu$, where sp denotes the canonical map from $\mathscr{B}$, the sheaf of hyperfunctions, to $\pi_{*} \mathcal{C}$, the direct image of the sheaf of microfunctions under the canonical projection $\pi: \sqrt{-1} S^{*} M \longrightarrow M$. As is well known, one of Sato's fundamental theorems on microfunctions asserts that

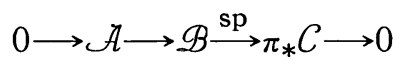

is exact, where $\mathcal{A}$ denotes the sheaf of real analytic functions (Sato [6]). In the sequel we sometimes abbreviate $\operatorname{sp}(\mu)$ to $\mu$, if there is no fear of confusions.

Using Theorem 6.4.1 in Sato [6], we easily find

$$
\text { S. S. }(S(w-x) E(x-y) u(y)) \subset A_{1} \cup A_{2} \text {, }
$$
where

$A_{1}=\{(x, y, w ; \sqrt{-1}(\xi, \eta, \zeta) \mid w-x \in L, y \in K, \xi=-\alpha \kappa+a \beta \vartheta$, $\eta=\beta(-a \theta+b \vartheta)$ and $\zeta=\alpha \kappa$ for some real non-zero n-dimensional co-vectors $\theta, \vartheta$ and $\kappa$ and non-negative constants $a$, $b, \alpha$ and $\beta$ satisfying $a+b \neq 0, \alpha+\beta \neq 0\}$

and

$A_{2}=\{(x, y, w ; \sqrt{-1}(\xi, \eta, \zeta) \mid w-x \in L, y \notin K, \xi=-\alpha \kappa+\beta \theta$, $\eta=-\beta \theta$ and $\zeta=\alpha \kappa$ for some real non-zero n-dimensional co-vectors $\theta$ and $\kappa$ and non-negative constants $\alpha$ and $\beta$ satisfying $\alpha+\beta \neq 0\}$. 
Here $(\xi, \eta, \zeta)$ denotes the homogeneous fiber coordinates of $S^{*} M$, where we denote $\boldsymbol{R}^{3 n}$ by $M$.

In the same way we can easily verify

S. S. $(S(w-x) E(y) u(x-y)) \subset B_{1} \cup B_{2}$, where

$B_{1}=\{(x, y, w ; \sqrt{-1}(\xi, \eta, \zeta) \mid x-y \in K, w-x \in L, \xi=-\alpha \kappa$ $+\beta b \theta, \eta=\beta(a \vartheta-b \theta)$ and $\zeta=\alpha \kappa$ for some real non-zero $n$ dimensional co-vectors $\theta, \vartheta$ and $\kappa$ and non-negative constants $a, b, \alpha$ and $\beta$ satisfying $a+b \neq 0, \alpha+\beta \neq 0\}$ $B_{2}=\{(x, y, w ; \sqrt{-1}(\xi, \eta, \zeta) \mid w-x \in L, \quad x-y \notin K, \quad \xi=\alpha \kappa$, $\eta=\beta \vartheta$ and $\zeta=-\alpha \kappa$ for some real non-zero $n$-dimensional co-vectors $\vartheta$ and $\kappa$ non-negative constants $\alpha$ and $\beta$ satisfying $\alpha+\beta \neq 0\}$.

Now, denoting $\boldsymbol{R}^{n}$ by $N$, we consider the smooth map $f: M \rightarrow N$ defined by $f(x, y, w)=w$. Then (8) and (9) immediately show that the canonical projection

$$
\sigma: \sqrt{-1} S^{*} N \times \underset{N}{\rightarrow} M \longrightarrow \sqrt{-1} S^{*} N,
$$

which is induced by $f$, turns out to be a proper map if is restricted to S.S. $(S(w-x) E(x-y) u(y))$ or S.S. $(S(w-x) E(y) u(x-y))$. In fact, by making full use of the non-zero assumptions on $a+b, \alpha+\beta$, $\theta, \vartheta$ and $\kappa$ given in (8) and (9), one can easily verify

$$
\sqrt{-1} S^{*} N \underset{N}{\times} M \cap A_{2}=\phi
$$

and

$$
\sqrt{-1} S^{*} N \underset{N}{\times} M \cap B_{2}=\phi .
$$

Then the required statements concerning the map $f$ is clear, since $K$ and $L$ are compact by the assumptions.

Therefore we can perform the following integration (12) along fiber, which makes sense as that of microfunctions (Sato [6] Corollary 6. 5. 3.):

$$
\int S(w-x) E(x-y) u(y) d y d x
$$


Then "the Fubini theorem" concerning the integration along fiber (Sato [2] Theorem 6.5.2) applies to the integral defined above and we find that it is equal to

$$
\int\left(\int S(w-x) E(x-y) d x\right) u(y) d y \text {. }
$$

Since

$$
\int S(w-x) E(x-y) d x=\delta(w-y)
$$

holds as the equality for hyperfunction by the choice of $E(x)$, the integral (13) of microfunctions is equal to $u(w)$.

On the other hand, using "the Fubini theorem" again, we find that the integral (12) is equal to

$$
\begin{aligned}
\int S(w-x)\left(\int E(x-y) u(y) d y\right) d x \\
=\int S(w-x)\left(\int E(y) u(x-y) d y\right) d x \\
=\int\left(\int S(w-x) u(x-y) d x\right) E(y) d y .
\end{aligned}
$$

Since

$$
\int S(w-x) u(x-y) d x=(S * u)(w-y)
$$

is a real analytic function of $w$ and $y$ by condition (3),

$$
\int\left(\int S(w-x) u(x-y) d x\right) E(y) d y
$$

vanishes as a microfunction. It immediately implies $u(w)$ vanishes identically as a microfunction, since the integral (14) is equal to the integral (13) and the integral (13) is equal to $u(w)$ as a microfunction. Then the exact sequence (7) proves that $u(w)$ is real analytic on $\boldsymbol{R}^{n}$. This completes the proof of the theorem.

Research Institute for Mathematical Sciences KYOTO UNIVERSITY

\section{References}

[1] Kawai, T.: The theory of Fourier transforms in the theory of hyperfunctions and its applications. Sûrikaisekikenkyusho Kôkyûroku No. 108, R. I. M.S. Kyoto Univ., pp. 84-288 (1970) (in Japanese).

[2] - On the theory of Fourier hyperfunctions and its applications to 
partial differential equations with constant coefficients. J. Fac. Sci. Univ. Tokyo, 17 (1970), 467-517.

[3] - On the propagation of analyticity of solutions of systems of linear differential equations with constant coefficients. To appear in J. Fac. Sci. Univ. Tokyo. (See also the technical report RIMS-90 of the Research Institute for Mathematical Sciences, Kyoto Univ. (1971).)

[4] Roumieu, Ch.: Sur quelques extensions de la notion de distributions. Ann. Sci. Ecole Norm. Sup. 77 (1960), 47-121.

[5] Sato, M.: Theory of hyperfunctions, II. J. Fac. Sci. Univ. Tokyo. 8 (1959), 387-437.

[6] - Structure of hyperfunctions. Sûgaku no Ayumi, 15 (1970), 9-72 (Notes by Kashiwara, in Japanese).

[7] Sato, M., T. Kawai and M. Kashiwara: Microfunctions and pseudo-differential equations. To appear in the report of Katata symposium. (Springer Lacture Notes No. 287) 